\title{
Ab initio study of clumped isotopic fractionation during acid digestion of dolomite $\left(\mathrm{CaMg}\left(\mathrm{CO}_{3}\right)_{2}\right)$
}

\author{
Chirantan PRAMANiK ${ }^{1}$, SANChitA BANERJEe ${ }^{2}$, \\ PROSENJIT GHOSH ${ }^{1,2}$ \\ ${ }^{1}$ Centre for Atmospheric and Oceanic Sciences, IISc \\ Bangalore, Bangalore 560012, chirantan88@gmail.com \\ ${ }^{2}$ Centre for Earth Sciences, IISc Bangalore, Bangalore \\ 560012, sanchita933@gmail.com \\ 3nghosh@iisc.ac.in
}

The basis of stable isotope paleothermometry is the clumped isotopic bonds $\left({ }^{13} \mathrm{C}-{ }^{18} \mathrm{O}\right)$ in carbonate mineral lattice. Carbonate is reacted with anhydrous phosphoric acid and product $\mathrm{CO}_{2}$ is used in mass spectrometer to determine $\Delta_{47}$, which is a measure of clumped isotope anomaly[1]. The process of digestion of carbonate involves loss of one oxygen atom from the $\mathrm{CO}_{3}{ }^{2-}$ radical to the product $\mathrm{CO}_{2}$. Theoretically Guo et al.,2009[2] derived acid digestion fractionation from $\mathrm{CO}_{3}{ }^{2-}$ to $\mathrm{CO}_{2}$ which is independent of the cation in the carbonate. In this study we perform ab initio calulations on acid digestion reaction of dolomite and predict the acid digestion fractionation amount.

Quantum chemical calculations are performed in Gaussian09[3] package with DFT-B3LYP level of theory and $6-311+\mathrm{G}(\mathrm{d}, \mathrm{p})$ basis set with water medium as background using self-consistent reaction field (SCRF) model. Transition state (TS) structures for protonation on dolomite are determined using 'qst2' and 'qst3' method implemented in Gaussian09 and confirmed by one negative frequency, The TS frequencies are used to calculate the acid digestion fractionation $\Delta_{47}$ using reduced partition function method.

Theoretical slope governing temperature $\left(10^{5} / \mathrm{T}^{2}\right)$ of reaction and acid fractionation $\Delta_{47}$ simulated in this study mimic the experimental condition observed in the common acid bath (CAB) method. The slope observed is 0.339 and is similar to the experimentally predicted slope value of 0.370 for $\mathrm{CAB}$ method[4]. However, where recations are conducted at low temperature $\left(25^{\circ} \mathrm{C}\right)$ and prologed time ( 72 hours) is best represented by the results of Guo et al.,2009 (slope is 0.189 ). The slope which we identifed experimentally for the sealed vessel (SV) method is 0.069 , which is not similar but close to the slope designated by Guo et al.,2009.

[1] P. Ghosh et al., Geochimica et Cosmochimica Acta 71 (2007) 2736-2744. [2] Weifu Guo et al., Geochimica et Cosmochimica Acta 73 (2009) 7203-7225. [3] M. J. Frisch et al. Gaussian 09, Revision A.02. [4] S. T. Murray et al., Geochimica et Cosmochimica Acta 174 (2016) 42-53. 